\title{
Resistively enhanced proton acceleration via high-intensity laser interactions with cold foil targets
}

\author{
Paul Gibbon* \\ John von Neumann Institute for Computing, Forschungszentrum Jülich GmbH, ZAM, D-52425 Jülich, Germany
}

(Received 13 May 2004; revised manuscript received 18 May 2005; published 25 August 2005)

\begin{abstract}
The acceleration of $\mathrm{MeV}$ protons by high-intensity laser interaction with foil targets is studied using a recently developed plasma simulation technique. Based on a hierarchical $N$-body tree algorithm, this method provides a natural means of treating three-dimensional, collisional transport effects hitherto neglected in conventional explicit particle-in-cell simulations. For targets with finite resistivity, hot electron transport is strongly inhibited, even at temperatures in the $\mathrm{MeV}$ range. This leads to suppression of ion acceleration from the rear of the target and an enhancement in energies and numbers of protons originating from the front.
\end{abstract}

DOI: 10.1103/PhysRevE.72.026411

PACS number(s): 52.38.Kd, 52.35.Mw, 52.65.Yy, 52.75.Di

\section{INTRODUCTION}

Ion acceleration using short-pulse, high-intensity lasers focused onto solid targets has received an increasing amount of experimental [1-4] and theoretical [5-7] attention over the last five years. This burgeoning interest stems from the huge potential offered by such compact, laser-based $\mathrm{MeV}$ protonion sources for applications in imaging [8], hadron therapy [9], isotope production [10], and nuclear fusion [11]. Of central importance for all of these applications is a quantitative knowledge of the ion beam characteristics, such as its energy spectrum, emittance, and source size, none of which can be effectively optimized without an understanding of the underlying physics governing the acceleration mechanisms.

To date, this phenomenon has been studied almost exclusively using particle-in-cell (PIC) simulations [5,7,12], which predict that the majority and/or most energetic protons originate from the rear of the target surface. This scenario relies on fast $(\mathrm{MeV})$ electrons created via nonlinear heating by the laser on the front of the irradiated target. These pass through the target, setting up a strong, static Debye sheath at the rear, thus pulling ions and proton deposits away from this surface [7].

Experimental support for this picture has been put forward by a number of groups [4,13], citing evidence that (i) protons tend to be emitted normal to the rear surface, even when the latter is at some angle to the front surface, and (ii) preheating the rear surface-thus cleaning it of water vapor and other hydrocarbon deposits-leads to a strongly reduced proton-ion signal. A sharply contrasting viewpoint has been championed by the Imperial College London, Michigan and lately Osaka groups $[3,14,15]$, who argue that the majority of $\mathrm{MeV}$ protons must originate from the front (laser-irradiated) side of the target via the collisionless shock driven by the ponderomotive force of the laser.

In view of the wide range of laser and target parameters used in these experiments, it is of course quite possible that both camps are "right" and that variable interaction conditions are bound to bias one mechanism in favor of the other,

*Electronic address: p.gibbon@fz-juelich.de as found in recent studies by the Jena and Garching groups $[16,17]$. The problem for the proponents of the front-side scenario is that the most comprehensive simulation tools available to date-three-dimensional (3D) electromagnetic PIC codes-all predict consistently higher rear-side proton energies and densities. Only at intensities $>5 \times 10^{20} \mathrm{~W} \mathrm{~cm}{ }^{2}$ and foil thicknesses $\leqslant 5 \mu \mathrm{m}$ does the front-side shock mechanism appear to dominate [18].

The purpose of this paper is to examine this issue using a fresh approach; a gridless $N$-body technique which, although still in a comparatively early stage of development, already overcomes some major limitations of collisionless PIC simulations, such as the necessity of resolving the electron Debye length for numerical stability and the almost unavoidable use of transverse-periodic boundary conditions. Moreover, the control over the plasma collisionality afforded by this method allows us to explore interactions starting from a more realistic, initially "cold" target state. In fact, it is demonstrated that besides the hot electron temperature, the electrical conductivity of the target plays a major role in determining where and to what energies protons are accelerated. This correlation between fast electron transport inhibition and ion dynamics is discussed later in Sec. IV: first, however, we introduce the concept of gridless collisional plasma modeling in Sec. II and give a brief outline of the laser model in Sec. III. Finally, some conclusions from this preliminary study are drawn in Sec. V.

\section{COLLISIONAL PLASMA SIMULATION USING A HIERARCHICAL TREE CODE}

The code used here, PEPC [19], is based on the BarnesHut hierarchical tree algorithm [20], implemented in parallel using the hashed oct-tree scheme of Warren and Salmon [21]. Briefly summarized: the electrostatic force sum on each particle is computed by systematically replacing more distant charges by multipole expansions of charge groups, thus reducing the standard $O\left(N^{2}\right)$ direct sum to an $O(N \log N)$ complexity at the price of a small, controllable error [22].

An earlier incarnation of this code was used to perform molecular dynamics (MD) calculations of nonlinear inverse bremsstrahlung absorption in strongly coupled plasmas 


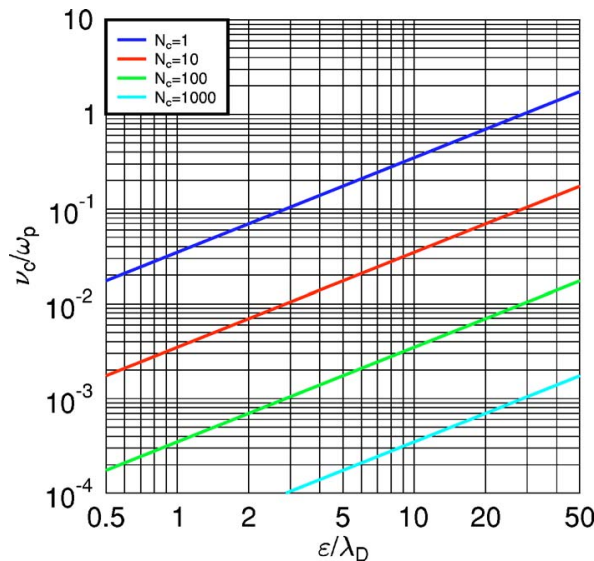

FIG. 1. (Color online) Normalized electron-ion collision frequency for finite-sized particles as a function of cloud radius for various values of $N_{c}$.

$[23,24]$. In the present context, PEPC is used in the macroscopic sense, using quasiparticles to trace the dynamics of phase-space elements just as in PIC simulation. The analogy with PIC stops there, however: first, the present tree code does away with both spatial grid and artificial boundaries completely; second, both electron-ion and electron-electron collisions are implicitly included in a natural, adjustable manner, drawing on the theoretical framework for "finitesize-particle" (FSP) simulations set out 30 years ago by Langdon and Birdsall [25] and Okuda and Birdsall [26].

According to this theory, the collisionality of a plasma comprising finite-sized clouds with radius $\varepsilon$ is typically reduced by orders of magnitude compared to a "real" plasma comprising point particles, so that the number of particles in a Debye sphere, $N_{D}=(4 \pi / 3) n \lambda_{D}^{3}$, is effectively replaced by the parameter $N_{c}=(4 \pi / 3) n \varepsilon^{3}$, where $n$, and $\lambda_{D}$ are the number density and Debye length, respectively. Okuda and Birdsall [26] expressed this attenuation effect quantitatively by evaluating the scattering cross section $\sigma_{\text {cloud }}$ for charge clouds numerically and then plotting the ratio $\sigma_{\text {cloud }} / \sigma_{\text {point }}$ as a function of the cloud radius and $N_{D}$.

In the large cloud limit $\varepsilon / \lambda_{D} \gg 1$ (the regime of interest for the present study), the curves in Fig. 7 of Ref. [26] can be fitted to better than $20 \%$ by a conveniently simple expression

$$
\frac{\sigma_{\text {cloud }}}{\sigma_{\text {point }}}=\frac{1}{3 \ln \Lambda}\left(\frac{\lambda_{D}}{\varepsilon}\right)^{2}
$$

where $\Lambda=9 N_{D}$. Applying the usual definition $\nu=\overline{n v} \sigma$, one can thus write down an effective collision frequency for cloud charges:

$$
\frac{\nu_{c}}{\omega_{p}} \simeq \frac{Z}{30 N_{D}}\left(\frac{\lambda_{D}}{\varepsilon}\right)^{2}=\frac{Z}{30 N_{c}}\left(\frac{\varepsilon}{\lambda_{D}}\right)
$$

This expression is also displayed graphically in Fig. 1. In obtaining this fit, we have also made use of the usual expression for point particles $[27,28]$ :

$$
\frac{\nu_{e i}}{\omega_{p}}=\frac{1}{3}\left(\frac{\pi}{32}\right)^{1 / 2} \frac{Z \ln \Lambda}{N_{D}} \simeq \frac{Z \ln \Lambda}{10 N_{D}},
$$

where $\omega_{p}$ is the plasma frequency.

In PIC codes the particle size is usually equivalent to the grid spacing $\Delta$, which must be kept $\lesssim \lambda_{D}$ to avoid aliasing instabilities, usually manifesting themselves as numerical heating [29]. To map the particle densities smoothly onto the grid, it is also desirable to have as many particles per cell as possible or $N_{c} \gg 1$ (although three-dimensional simulations are often performed with as few as two to three electrons/ ions per cell). This combination means that PIC codes are typically operated in the bottom-left, "collisionless" corner of Fig. 1, with $\nu_{c} / \omega_{p} \leqslant 10^{-2}$.

By contrast, the gridless FSP approach gives us the freedom to set up a simulation within a much larger area of the parameter space depicted in Fig. 1. The most sensitive parameter here is $N_{c}$ or, equivalently, $\varepsilon / \bar{a}$, the ratio of the cloud size to the average interparticle spacing. Even at modest densities, adjusting $\varepsilon / \bar{a}$ enables us to emulate both hot, collisionless plasmas where PIC simulation is valid or the cold, collisional state normally treated with hydrodynamic or Fokker-Planck modeling. Choosing a large $\varepsilon / \lambda_{D}$ (or low temperature) will wash out details on the cold electron Debye-length scale (such as Langmuir wave dispersion), but this is of minor concern for problems where the physics is dominated by large- (micron-) scale charge separation effects.

In practical terms, the finite cloud size is introduced via a smoothed Coulomb potential, which in PEPC takes the form

$$
\boldsymbol{\Phi}(\boldsymbol{r})=\frac{q \boldsymbol{r}}{\left(r^{2}+\varepsilon^{2}\right)^{1 / 2}},
$$

with the corresponding equation of motion for each particle $i$

$$
m_{i} \frac{d \boldsymbol{u}_{i}}{d t}=\frac{1}{3} q_{i} \sum_{i \neq j} \frac{q_{j} \boldsymbol{r}_{i j}}{\left(r_{i j}^{2}+\varepsilon^{2}\right)^{3 / 2}}+q_{i} \boldsymbol{E}^{p}\left(\boldsymbol{r}_{i}\right),
$$

where $\boldsymbol{r}_{i j}=\boldsymbol{r}_{i}-\boldsymbol{r}_{j}$ is the separation between particles $i$ and $j$, $\boldsymbol{u}_{i}=\gamma \boldsymbol{v}_{i}$ is its proper velocity with relativistic factor $\gamma=(1$ $\left.+|\boldsymbol{u}|^{2} / c^{2}\right)^{1 / 2}$, and $\boldsymbol{E}^{p}$ is an external field arising from the laser (see Sec. III).

In Eqs. (4) and (5) the variables $t, r, v, q, m, \Phi$, and $E$ have been normalized to $\omega_{p}^{-1}, c \omega_{p}^{-1}, N_{p} e, N_{p} m_{e}, m_{e} c^{2} / e$, and $m_{e} \omega_{p} c / e$, respectively, where

$$
N_{p}=\frac{4 \pi}{3} n_{e}\left(\frac{c}{\omega_{p}}\right)^{3}
$$

is a dimensionless constant representing the number of physical charges contained within a simulation particle.

To set up a FSP simulation with PEPC, the electrons and ions are first brought into a homogeneous, equilibrium configuration according to the target geometry, density $n_{0}$, and electron temperature $T_{e}$ [30]. The foil targets considered here have dimensions $L_{x} L_{y} L_{z}=5 \times 12 \times 12 \mu \mathrm{m}^{2}$, comprising 3.2 $\times 10^{6}$ electrons and ions, giving $\bar{a}=0.7 c / \omega_{p}$. The electron and ion densities are initially set to $n_{i}=n_{e}=n_{0}=(4 \rightarrow 10) n_{c}$, where $n_{c}$ is the critical density corresponding to the laser 
frequency $\omega$, related by $\omega^{2}=4 \pi e^{2} n_{c} / m_{e}$, where $e$ and $m_{e}$ are the electronic charge and mass, respectively. The ions are given a charge $Z=1$, mass $m_{i}=1836 m_{e}$, and initial temperature $T_{i}=0$. Unlike in PIC codes, the electron temperature is not artificially constrained to some value (typically several $\mathrm{keV}$ ) determined by the mesh size, but can be varied from a few 10's of eV upwards to control the initial target resistivity. For lower temperatures, however, the time step [typically $\left.(0.1-0.5) \omega_{p}^{-1}\right]$ is generally reduced in order to resolve the collision dynamics and ensure reasonable energy conservation.

\section{LASER MODEL}

The laser is modeled using a ponderomotive standing wave ansatz for the electromagnetic field $E_{L}$ applied at the vacuum-plasma boundary on the front side of the target, a more detailed description of which can also be found elsewhere [30]. Essentially the laser field is represented by a relativistic potential

$$
\gamma=(1+\Psi)^{1 / 2}
$$

where

$$
\Psi=4 a_{0}^{2} X^{2}(x) R(r) T(t) .
$$

The longitudinal, radial, and temporal components in the above expression are, respectively, given by

$$
\begin{gathered}
X(x)= \begin{cases}\sin \chi, & x<0, \\
\sin \phi \exp \left(-x / l_{r}\right), & x \geqslant 0,\end{cases} \\
R(r)= \begin{cases}\cos ^{2}\left(\frac{\pi r}{4 \sigma}\right), & r \leqslant 2 \sigma, \\
0, & r>2 \sigma,\end{cases} \\
T(t)=\sin ^{2}(\omega t),
\end{gathered}
$$

where $\omega$ is the laser frequency normalized to $\omega_{p}$. The spatial terms are phase matched at the boundary (or critical surface) $x=x_{c}=0$ by choosing

$$
\begin{gathered}
\phi=-\tan ^{-1}\left(k l_{r}\right), \\
\chi=k x+\phi,
\end{gathered}
$$

where $k$ is the laser wave number normalized to $\omega_{p} / c$. The skin depth $l_{s}=c / \omega_{p}$ has been modified to correct for enhanced penetration of the evanescent wave at relativistic pump strengths $a_{0}=e E_{L} / m_{e} \omega c>1$ [31], so that

$$
l_{r}=\gamma_{s}^{1 / 2} l_{s}
$$

where

$$
\gamma_{s}=\left(1+4 a_{0}^{2} n_{c} / n_{u}\right)^{1 / 2}
$$

corresponds to the field amplitude at the interface $x=0$ and $n_{u}$ is an averaged upper shelf density (initially equal to $n_{0}$ ).

The radius $r=\left(y^{2}+z^{2}\right)^{1 / 2}$ is taken relative to the center of the focal spot. This form is used in order to create a sharp radial cutoff at $r=2 \sigma_{L}\left(\sigma_{L}\right.$ is the half width at half maximum). The time-dependent component $T(t)$ provides both the $j \times B$ heating and dc push on the electron density. Finally, the longitudinal and radial ponderomotive field components (applied as external forces in the momentum equation for the electrons) are found from $E_{x}^{p}=d \gamma / d x$ and $E_{r}^{p}=d \gamma / d r$, respectively [30].

Despite its obvious simplicity, this model exhibits surprisingly good agreement with one-dimensional, electromagnetic PIC simulations in terms of the field structure, fast electron heating, and ion shock dynamics, provided the electron density scale length $L$ remains small compared to the laser wavelength $\lambda$ [30]. For high laser intensities, the profile is typically steepened and driven forwards, so that we also need to track the critical surface when computing the laser fields. This approach is similar in spirit to that used in hydrodynamic and Fokker-Planck simulations, incorporating the laser via an adaptive heat and momentum source. An important difference in this model, however, is that the electrons are accelerated self-consistently by the laser field and/or plasma oscillations: there is no need for an ad hoc prescription for the hot electron temperature. The most serious omission here is the absorption of electromagnetic radiation by the plasma (pump depletion), so that the reflectivity and net field intensity in the vacuum region will be overestimated if the absorption fraction is high. To remedy this, one could include appropriate corrections to Eqs. (7)-(9) (or ideally solve the full three-dimensional Helmholtz equations for the laser field), but refinements such as these are well beyond the scope of the present study.

\section{PROTON ACCELERATION: HOT vs COLD TARGETS}

The electrical conductivity of the target, treated here assuming a classical, pre-ionized plasma, determines the magnitude of the return current which can be supplied by the cold background electrons. Previous theoretical [32] and experimental [33] work has demonstrated that resistive effects already inhibit hot electron penetration for intensities as low as $10^{17} \mathrm{~W} \mathrm{~cm}^{-2}$. The Spitzer resistivity can be related to the effective collision frequency $\widetilde{\nu}_{e i} \equiv \nu_{c} / \omega_{p}$ used in the model [Eq. (2)] simply via

$$
\begin{aligned}
& \eta_{e}=\frac{m_{e} \nu_{e i}}{n_{e} e^{2}}=\frac{1}{\omega_{p} \varepsilon_{0}} \widetilde{\nu}_{e i} \quad(\mathrm{SI}) \\
& =6.3 \times 10^{-6} n_{23}^{-1 / 2} \widetilde{\nu}_{e i} \Omega \mathrm{m},
\end{aligned}
$$

where $n_{23}$ is the electron density in units of $10^{23} \mathrm{~cm}^{-3}$.

For example, to emulate the resistivities of $\sim 200 \mu \Omega \mathrm{cm}$ measured by Milchberg et al. [34] for Al targets, we would need to set up a model plasma with $\widetilde{\nu}_{e i} \sim 0.3$. On the other hand, the actual collision frequency for these experimental conditions $\left(n_{23}=1, T_{e}=20 \mathrm{eV}, Z=3\right)$ is $\nu_{e i} / \omega_{p}=0.8$, so that the model and "real" targets can easily be matched up in this case. Spitzer resistivity will of course apply neither to insulators nor to extremely dense, cold (strongly coupled) plasmas: in the remainder of the paper we will therefore simply treat $\widetilde{\nu}_{e i}$ as a free parameter with which to vary the collision- 

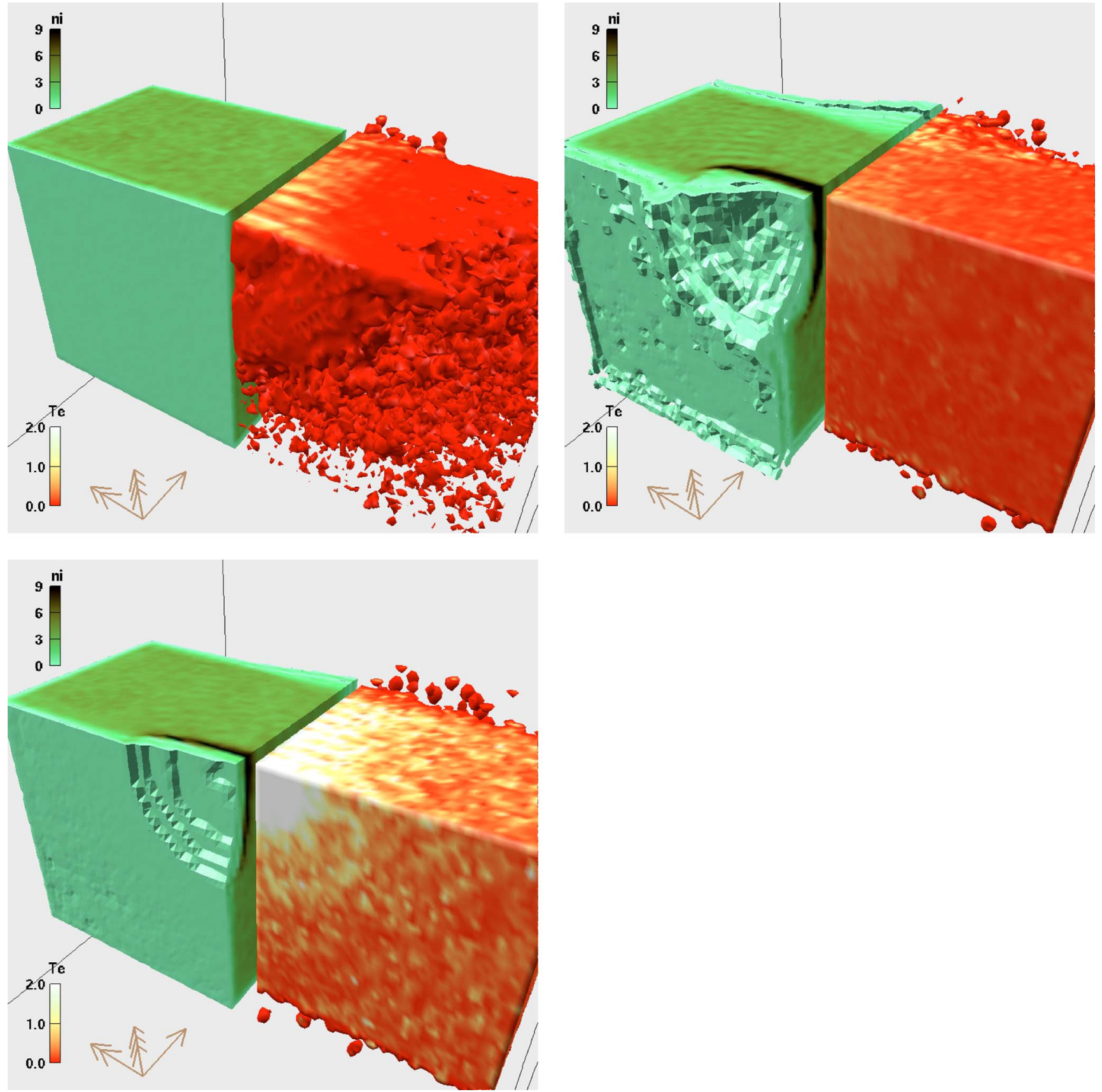

FIG. 2. (Color online) Isovolume sequences of ion density (left, threshold $n_{c} / 20$ ) and mean electron energy (right, threshold $U_{h} \geqslant 10 \mathrm{keV}$ ) sliced half-way through the target in the $x z$ plane for a target with initial normalized resistivity of $\bar{\eta}_{e}=7 \times 10^{-3}$. Snapshots are taken at times $\omega_{p} t=$ (a) 100 (26 fs), (b) 350 (92 fs), and (c) 650 (170 fs).

ality rather than attempt any kind of realistic target modelling.

To determine how the inhibition of electron transport affects ion acceleration, we compare two simulations with different target conductivities but otherwise identical parameters: $\quad I \lambda^{2}=2.5 \times 10^{19} \mathrm{~W} \mathrm{~cm}^{-2} \mu \mathrm{m}^{2}\left(a_{0}=4\right), \quad \sigma_{L}=15 \mathrm{c} / \omega_{p}$, (square) pulse duration $\tau_{L}=100 \mathrm{fs}$, and initial plasma density $n_{0} / n_{c}=4$. The initial electron temperatures in the two cases are $5 \mathrm{keV}$ and $500 \mathrm{eV}$; the particle diameters $\varepsilon=3$ and 0.7 , giving effective normalized resistivities $\widetilde{\eta}_{e} \equiv \widetilde{\nu}_{e i}=7 \times 10^{-3}$ and 0.45 , respectively. For a solid $\mathrm{Al}$ target $\left(n_{23}=1\right)$, these values would correspond to initial resistivities of $4.4 \mu \Omega \mathrm{cm}$ and $280 \mu \Omega \mathrm{cm}$, respectively.

In the high-temperature case, the effective hot electron range determined by electrostatic stopping is [32] $R_{h}$ $\approx 80 \mu \mathrm{m}$, so we expect the simulation to behave much like a collisionless PIC code would. This is just what we observe in Fig. 2, which shows three-dimensional snapshots of the ion density and hot electron temperature. This plot encapsulates many of the salient features of high-intensity interactions familiar from 2D and 3D PIC simulations to date: bursts of $(j \times B)$-accelerated electrons generated at $2 \omega$ freely travers- 

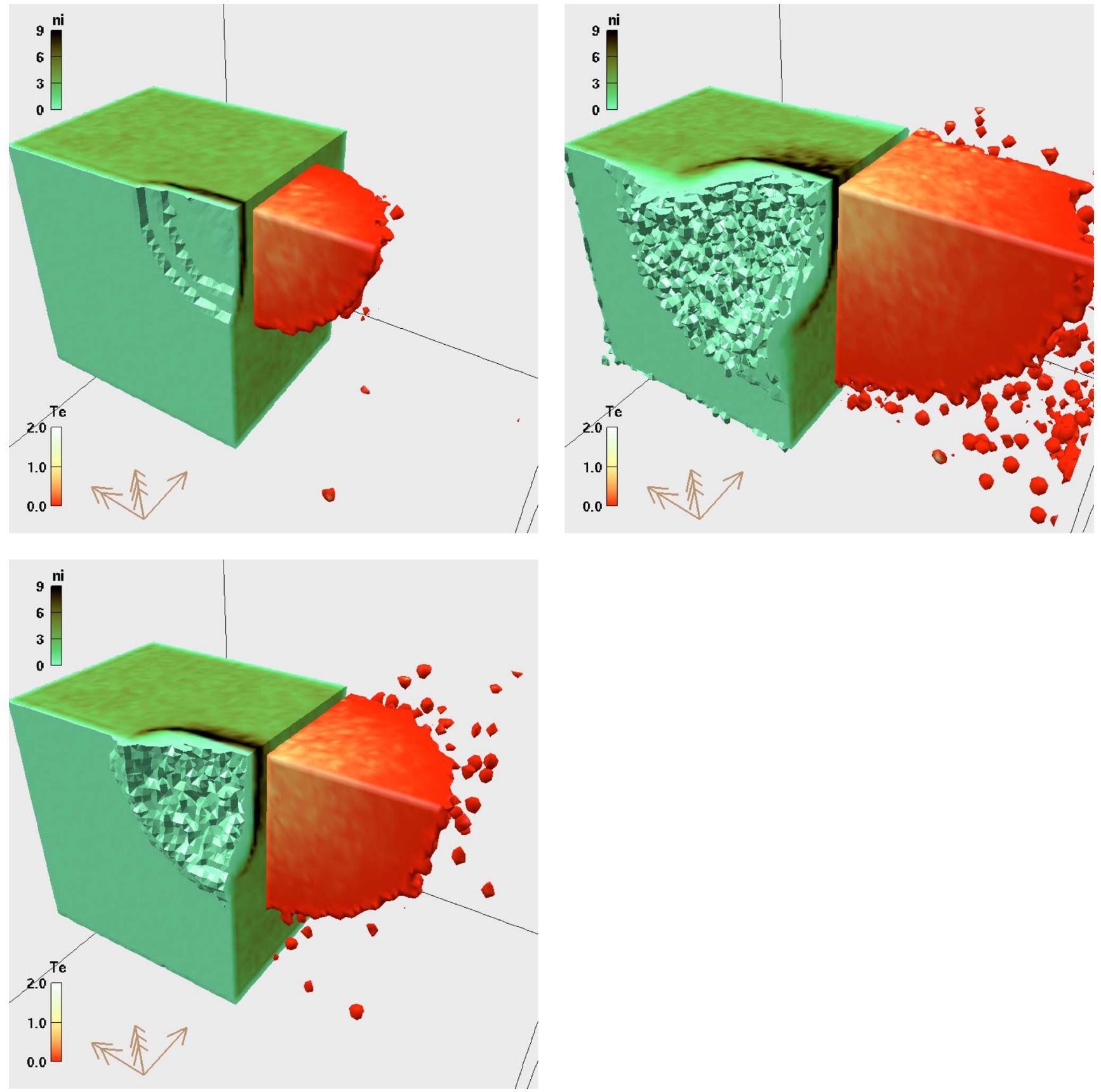

FIG. 3. (Color online) As Fig. 2, but for a target with initial normalized resistivity of $\bar{\eta}_{e}=0.45$. Snapshots are taken at times $\omega_{p} t=($ a) 180 (48 fs), (b) 360 (95 fs), and (c) 650 (170 fs).

ing the target, formation of a ponderomotively driven ion shock on the front side, and a hot electron Debye sheath being formed on the rear side, pulling ions away from the surface. We also find that the whole foil has been heated to over $50 \mathrm{keV}$ in under $100 \mathrm{fs}$, in agreement with the 3D PIC simulation in Ref. [5].

Compare this now with Fig. 3, a similar sequence for the $500 \mathrm{eV}$ "resistive" simulation, for which the hot electron range is now $R_{h} \approx 1.2 \mu \mathrm{m}$ [see Eq. (12) below]. This time we see a completely different picture: despite having energies in the $\mathrm{MeV}$ range, the hot electrons are confined to a hemispherical heat front, 1-2 $\mu \mathrm{m}$ ahead of the shock, and are virtually absent from the rear-side vacuum region at this time. This is consistent with the analytical model of Bell et al. [32] and the 2D Fokker-Planck simulations of Davies et al. [35], which predict a diffusive rather than free-streaming behavior at intensities high enough to induce electrostatic transport inhibition.

These contrasting pictures are illustrated in more detail in Fig. 4, which shows snapshots of the electron kinetic energy along the laser axis, averaged over half the spot size. In Fig. 4(a), we again see the free-streaming behavior of $(j \times B)$-heated electrons; the heat front traversing the foil with velocity $u_{f} \approx c$. In the collisional case of Fig. 4(b), this velocity is drastically reduced to $u_{f} \sim 0.03 c$, considerably delaying the heating of the whole foil as a result. 

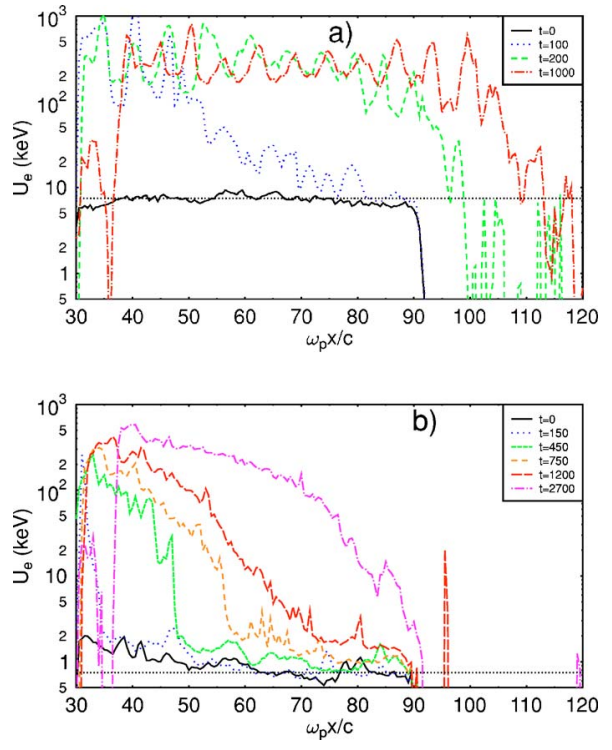

FIG. 4. (Color online) Lineouts of electron kinetic energy at different times during the interaction corresponding to (a) the collisionless case in Fig. 2 and (b) collisional case in Fig. 3. The straight dotted line at the bottom corresponds to the initial plasma temperature $\left(3 / 2 k_{B} T_{e}\right)$.

One could argue that the strong heat-flow inhibition seen in Fig. 4(b) is an artifact of the FSP ansatz, which in this instance imposes an "exaggerated" collisionality on a model plasma which would normally be collisionless, having a density $n_{e} / n_{c}=4$ and temperature $T_{e} \simeq 1 \mathrm{keV}$ during the simulation. Although this model is set up to emulate more realistic initial conditions $n_{e} / n_{c}=100, T_{e} \simeq 10-20 \mathrm{eV}$, these are subject to change (particularly heating) during the simulation. Once electrostatic inhibition is established, however, preheating of the cold plasma by hot electrons downstream of the heat front will be sharply reduced-in both "model" and "real" targets. Behind the heat front, the temperature in both simulations increases by around two orders of magnitude and the plasma reverts to a collisionless state. This behavior is consistent with that observed in previous Fokker-Planck simulations [35], albeit at lower intensities $\left(I \sim 10^{18} \mathrm{~W} \mathrm{~cm}^{-2}\right)$. The physics of electrostatic transport inhibition is therefore well reproduced by the FSP model, even if computational cost prevents us from matching the realism and resolution of Fokker-Planck codes at this time. On the other hand, the present approach has the advantage that no restriction is placed on the relative number densities of hot and cold electrons or distortion of the velocity distribution (and therefore laser intensity), both of which can cause numerical difficulties in FP codes.

The consequences of hot electron transport inhibition for the proton acceleration are dramatic: the absence (or significantly delayed presence) of the hot Debye sheath on the rear side clearly suppresses ion acceleration there. On the other hand, the resistively induced electric field in front of the shock will act to enhance the front-side acceleration. These observations are summarized in Fig. 5, which shows how the relative maximum energy of protons originating from the front and rear of the foil, respectively, reverses as the target resistivity is increased.

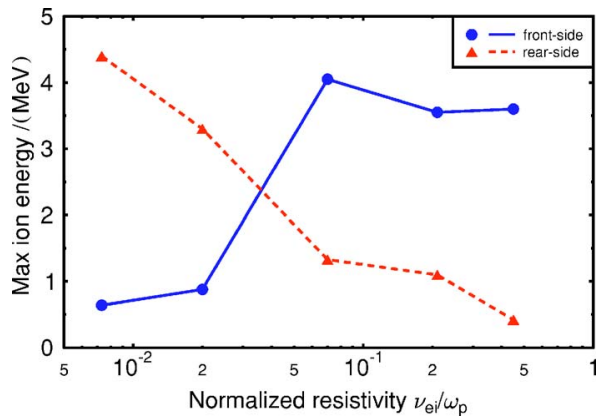

FIG. 5. (Color online) Maximum energy in $\mathrm{MeV}$ of protons originating from the front (solid line) and rear (dashed line) of the foil at $150 \mathrm{fs}$ as a function of target resistivity.

Each of the simulations in Fig. 5 took between 60 and $150 \mathrm{~h}$ on 32 processors of the Jülich IBM p690+Regatta, so that a comprehensive parameter study with the present code is still beyond reach. To provide some guidance for future studies and experiments, we therefore consider the scaling of proton energies with laser intensity and target thickness in this hitherto unexplored resistive regime. A criterion for suppression of rear-side ion acceleration can be estimated from Bell's analytical (one-dimensional) transport model [32], which for a square laser pulse predicts a linearly growing hot electron density of the form

$$
n_{h}(x, t)=n_{e} \frac{t}{t_{\mathrm{sat}}} \frac{R_{h}^{2}}{\left(x+R_{h}\right)^{2}},
$$

where $R_{h}=3 \sigma_{e} T_{h}^{2} / I_{a}$ and $t_{\mathrm{sat}}=9 \sigma_{e} T_{h}^{3} / 2 I_{a}^{2}$. These relations are expressed in terms of normalized variables, with $T_{h}$ in $m_{e} c^{2}$ and $I_{a}=\eta_{a} I_{L}$, the "absorbed" laser intensity, in $n_{e} m_{e} c^{3}$, so that $I_{a}=\frac{1}{2} \eta_{a}\left(n_{c} / n_{e}\right) a_{0}^{2}$. Assuming that the laser pulse is long enough, the hot electron component will eventually saturate $\left(n_{h} \sim n_{e}\right)$ in a time $O\left(t_{\text {sat }}\right)$. Moreover, we may define a hot electron traversal time across the foil by setting $n_{h}\left(d, t_{\text {foil }}\right)$ $=n_{c}$, at which point the charge buildup beyond the rear surface should be sufficient to be felt by the ions. Rearranging, gives

$$
t_{\text {foil }}=t_{\mathrm{sat}} \frac{n_{c}}{n_{e}}\left(\frac{d}{R_{h}}+1\right)^{2} .
$$

Front-side ion acceleration will be favored if $t_{\text {foil }}>t_{L}>t_{\text {sat }}$, simultaneously ensuring that $n_{h}(x=0) \gg n_{c}$ and that no hot electrons reach the rear surface, a condition which is satisfied for

$$
R_{h}<\frac{d}{\omega_{p} / \omega+1} .
$$

These criteria are clearly sensitive to the absorption physics; in particular to the conversion efficiency of laser energy to hot electrons, $\eta_{a}$, and the scaling of $T_{h}$ with $I_{L} \lambda^{2}$, both of which depend on the plasma density and scale length at the front surface [36]. An extensive preplasma will tip the balance back in favor of rear-side acceleration, since the return current can then be supplied from the hot, underdense region surrounding the focal spot. 

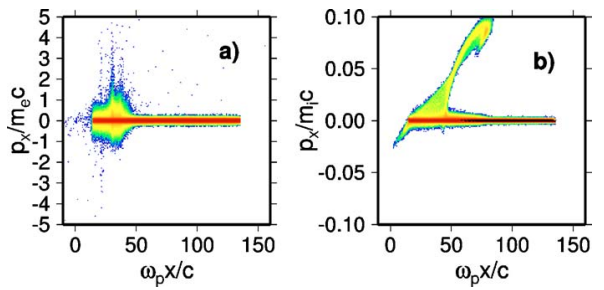

FIG. 6. (Color online) Resistive proton acceleration at $10^{20} \mathrm{~W} \mathrm{~cm}^{-2} \mu \mathrm{m}^{2}$ : (a) electron longitudinal phase space $\left(p_{x}, x\right)$ at the peak of a $75 \mathrm{fs}$ pulse and (b) ion phase space after the laser pulse at $\omega_{p} t=990$.

Hot electron penetration could also be enhanced by the focusing effects of self-generated magnetic fields, not yet included in this electrostatic model. As shown recently by Bell and Kingham [37], this magnetic field can reduce the solid angular spread of an initially isotropic hot electron "spray"-at least for the investigated parameter range [Terawatt powers, pulse durations of several hundred fs]. This effect will ultimately increase $R_{h}$ and reduce $t_{\text {foil }}$ accordingly. The interplay between magnetic fields and resistive effects at truly relativistic intensities still remains largely unexplored, however, and a proper assessment of this effect within the present model must await the future inclusion of magnetic fields.

Generally, for a fixed set of laser parameters, rear-side sheath acceleration will occur more readily for thin foils, assuming that the contrast ratio is sufficiently high to avoid significant rear-side ablation. For cold, thick targets, only the front-side mechanism will be active: exactly the opposite conclusion, in fact, of the purely collisionless PIC studies performed to date $[5,6,18]$. This is illustrated by another set of simulations with parameters $d=10 \mu \mathrm{m}, n_{e} / n_{c}=10, T_{e}$ $=500 \mathrm{eV}, \sigma_{e}=2, \varepsilon=0.64$, and a total of $6.72 \times 10^{6}$ particles, ensuring that the above criteria are comfortably met. This time a more realistic $\sin ^{2}$ pulse shape is used with $\omega_{p} t_{L}$ $=450[75 \mathrm{fs}$ full width at half maximum (FWHM)]. As expected, the hot electrons do not reach the rear side of the target and proton acceleration is confined to the front sideFig. 6.

An attractive property of protons accelerated in tow of the hot electron front is that they have a low-energy spreadFig. 7. This is reminiscent of the rear-side proton layer scheme proposed by Esirkepov et al. [38], again based on collisionless PIC simulation at $10^{21} \mathrm{~W} \mathrm{~cm}^{-2}$. By contrast, the present study suggests that laser-driven proton sources could be optimized at much lower intensities by placing a thin hydrocarbon layer (or other proton-loaded coating) onto the

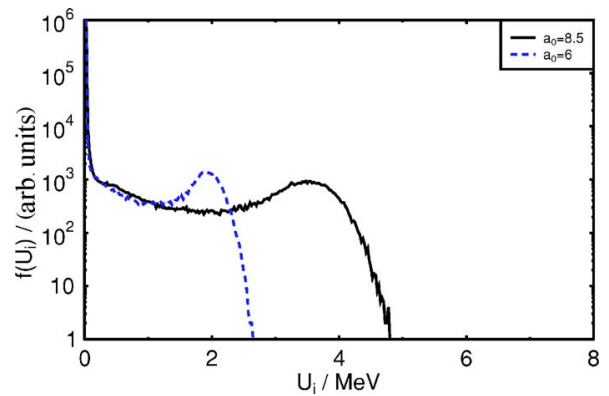

FIG. 7. Ion energy spectra showing beamlike emission from front-side protons. In these examples, no protons were emitted from the target rear surface.

front side of an insulating substrate, as indeed demonstrated recently by Zepf et al. [14].

\section{CONCLUSIONS}

In summary, simulations of proton acceleration in highintensity laser-solid interactions have been presented using a new type of mesh-free plasma model which permits both collective and collisional effects to be treated selfconsistently. This approach reintroduces the "finite-sizeparticle" ansatz examined 30 years ago in order to quantify the heavily reduced collisionality typically found in PIC simulations. A key advantage of the FSP technique is that the spatial grid can be dispensed with, thereby avoiding the numerical heating instability commonly associated with PIC simulations. As a result, "cold" plasma simulation becomes possible; the electron temperature playing a physically prominent role in determining the collisionality, both spatially and temporally.

As in previous studies of hot electron transport using analytical and numerical Fokker-Planck approaches, it is confirmed that a finite target resistivity leads to strong suprathermal heat-flow inhibition, even at intensities up to $10^{20} \mathrm{~W} \mathrm{~cm}^{-2}$. This in turn radically alters the nature of ion acceleration from foil targets, suppressing the sheath mechanism at the rear surface of the target and enhancing the shock-driven process at the front. These findings are in stark contrast to the predictions of particle-in-cell simulations to date and offer a plausible explanation for the dominant "front-side" proton emission observed in a number of recent laser interaction experiments.

\section{ACKNOWLEDGMENTS}

The author acknowledges valuable discussions with A. R. Bell concerning hot electron transport and a generous allocation of computing resources from the VSR Commission, FZ-Jülich, Project No. JZAM04.
[1] E. L. Clark et al., Phys. Rev. Lett. 84, 670 (2000).

[2] A. J. Mackinnon, M. Borghesi, S. Hatchett, M. H. Key, P. K. Patel, H. Campbell, A. Shiavi, R. Snavely, S. C. Wilks, and O. Willi, Phys. Rev. Lett. 86, 1769 (2001).

[3] A. Maksimchuk, S. Gu, K. Flippo, D. Umstadter, and V. Y.
Bychenkov, Phys. Rev. Lett. 84, 4108 (2000).

[4] R. A. Snavely et al., Phys. Rev. Lett. 85, 2945 (2000).

[5] A. Pukhov, Phys. Rev. Lett. 86, 3562 (2001).

[6] Y. Sentoku, T. E. Cowan, A. Kemp, and H. Ruhl, Phys. Plasmas 10, 2009 (2003). 
[7] S. C. Wilks et al., Phys. Plasmas 8, 542 (2001).

[8] M. Borghesi, A. J. Mackinnon, D. H. Campbell, D. G. Hicks, S. Kan, P. K. Patel, D. Price, L. Romagnani, A. Shiava, and O. Willi, Phys. Rev. Lett. 92, 055003 (2004).

[9] S. V. Bulanov et al., Phys. Lett. A 299, 240 (2002).

[10] K. Nemoto et al., Appl. Phys. Lett. 78, 595 (2001).

[11] M. Roth et al., Phys. Rev. Lett. 86, 436 (2001).

[12] H. Ruhl et al., Plasma Phys. Rep. 27, 387 (2001).

[13] M. Hegelich et al., Phys. Rev. Lett. 89, 085002 (2002).

[14] M. Zepf et al., Phys. Rev. Lett. 90, 064801 (2003).

[15] H. Habara, R. Kodama, Y. Sentoku, N. Izumi, Y. Kitagawa, K. A. Tanaka, K. Mima, and T. Yamanaka, Phys. Rev. E 69, 036407 (2004).

[16] S. Karsch, S. Düsterer, H. Schwoerer, F. Ewald, D. Habs, M. Hegelich, G. Pretzler, A. Pukhov, K. Witte, and R. Sanerbrey, Phys. Rev. Lett. 91, 015001 (2003).

[17] M. Kaluza, J. Schreiber, M. I. K. Santala, G. D. Tsakiris, K. Eidmann, J. Meyer-ter-Vehn, and K. J. Witte, Phys. Rev. Lett. 93, 045003 (2004).

[18] L. O. Silva, M. Marti, J. R. Davies, R. A. Fonseca, C. Ron, F. Tsung, and W. B. Mori, Phys. Rev. Lett. 92, 015002 (2004).

[19] P. Gibbon, W. Frings, S. Dominiczak, and B. Mohr, in Proceedings of the Parallel Computing Conference, Malaga, Sept 2005 (to be published).

[20] J. Barnes and P. Hut, Nature (London) 324, 446 (1986).

[21] M. S. Warren and J. K. Salmon, Comput. Phys. Commun. 87, 266 (1995).
[22] S. Pfalzner and P. Gibbon, Many Body Tree Methods in Physics (Cambridge University Press, New York, 1996).

[23] S. Pfalzner and P. Gibbon, Comput. Phys. Commun. 79, 24 (1994).

[24] S. Pfalzner and P. Gibbon, Phys. Rev. E 57, 4698 (1998).

[25] A. B. Langdon and C. K. Birdsall, Phys. Fluids 13, 2115 (1970).

[26] H. Okuda and C. K. Birdsall, Phys. Fluids 13, 2123 (1970).

[27] W. L. Kruer, The Physics of Laser Plasma Interactions (Addison-Wesley, New York, 1988).

[28] J. D. Huba, NRL Plasma Formulary (Naval Research Laboratory, Washington, DC, 1994).

[29] C. Birdsall and A. Langdon, Plasma Physics via Computer Simulation (McGraw-Hill, New York, 1985).

[30] P. Gibbon et al., Phys. Plasmas 11, 4032 (2004).

[31] P. Kaw and J. Dawson, Phys. Fluids 13, 472 (1970).

[32] A. R. Bell, J. R. Davies, S. Guérin, and H. Ruhl, Plasma Phys. Controlled Fusion 39, 653 (1997).

[33] D. Batani et al., Phys. Rev. E 65, 066409(2002).

[34] H. M. Milchberg, R. R. Freeman, S. C. Davey, and R. M. More, Phys. Rev. Lett. 61, 2364 (1988).

[35] J. R. Davies, A. R. Bell, M. G. Haines, and S. M. Guérin, Phys. Rev. E 56, 7193 (1997).

[36] P. Gibbon and A. R. Bell, Phys. Rev. Lett. 68, 1535 (1992).

[37] A. R. Bell and R. J. Kingham, Phys. Rev. Lett. 91, 035003 (2003).

[38] T. Z. Esirkepov et al., Phys. Rev. Lett. 89, 175003 (2002). 